\title{
Predicting the onset of psychosis in patients at clinical high risk: practical guide to probabilistic prognostic reasoning
}

\author{
P Fusar-Poli, ${ }^{1,2}$ F Schultze-Lutter ${ }^{3}$
}

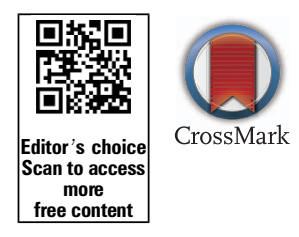

${ }^{1}$ King's College London, Institute of Psychiatry, Psychology and Neuroscience, London, UK; ${ }^{2}$ OASIS service, South London and the Maudsley NHS Foundation Trust, London, UK; ${ }^{3}$ University Hospital of Child and Adolescent Psychiatry and Psychotherapy, University of Bern, Bern, Switzerland

Correspondence to Dr P Fusar-Poli, Department of Psychosis Studies, Institute of Psychiatry PO63, De Crespigny Park, London SE58AF, UK; paolo.fusar-poli@kcl.ac.uk

\section{ABSTRACT}

Prediction of psychosis in patients at clinical high risk (CHR) has become a mainstream focus of clinical and research interest worldwide. When using CHR instruments for clinical purposes, the predicted outcome is but only a probability; and, consequently, any therapeutic action following the assessment is based on probabilistic prognostic reasoning. Yet, probabilistic reasoning makes considerable demands on the clinicians. We provide here a scholarly practical guide summarising the key concepts to support clinicians with probabilistic prognostic reasoning in the CHR state. We review risk or cumulative incidence of psychosis in, person-time rate of psychosis, Kaplan-Meier estimates of psychosis risk, measures of prognostic accuracy, sensitivity and specificity in receiver operator characteristic curves, positive and negative predictive values, Bayes' theorem, likelihood

ratios, potentials and limits of real-life applications of prognostic probabilistic reasoning in the CHR state. Understanding basic measures used for prognostic probabilistic reasoning is a prerequisite for successfully implementing the early detection and prevention of psychosis in clinical practice. Future refinement of these measures for CHR patients may actually influence risk management, especially as regards initiating or withholding treatment.

\section{INTRODUCTION}

Psychoses, in particular schizophrenia, are still one of the most costly and disabling disorders, despite their low incidence of 0.032 per 100 person-years (95\% Cl from 0.025 to 0.041 per 100 person-years). ${ }^{1}$ One cause of poor outcome of psychosis is their often long delay in adequate treatment initiation which his worsening the long-term general symptomatic outcome, positive and negative symptoms, likelihood of remission, social functioning and global outcome. ${ }^{2}$ Thus, over the past two decades, increasing efforts have been made to detect and treat psychosis early, preferably already during its prodromal phase of often many years in order to reduce the duration of untreated psychosis and improve the clinical outcomes. ${ }^{3}$ To an indicated prevention of psychosis in persons seeking help already for mental problems, two alternative clinical high risk (CHR) approaches to an early detection were developed: (1) the ultra-high risk (UHR) approach including attenuated psychotic symptoms (APS), brief limited intermittent psychotic symptoms or brief intermittent psychotic symptoms (BLIPS/BIPS) as well as a combination of genetic risk and functional decline (GRFD) and (2) the basic symptom (BS) approach based on subjective, subtle cognitive and perceptive disturbances that form two partly overlapping criteria. ${ }^{4}$ Both approaches (BS and UHR) as well as the instruments specifically developed for their assessment have shown the ability to detect a considerably increased CHR for psychosis with pooled 13 -year conversion rates to psychosis ranging from $15 \%$ to $29 \%$ for $\mathrm{UHR}^{5}{ }^{6}$ and from $14 \%$ to $50 \%{ }^{7}$ for BS criteria, and sufficient prognostic accuracy of their assessment, in particular in ruling out psychosis risk. ${ }^{8}$ However, these figures are not stable but were shown to vary not only with the follow-up time but also with characteristics of the group in which CHR state is assessed, for example, with age composition $^{9}$ or main referral source. ${ }^{8}$ Thus, when using these CHR criteria or instruments for clinical purposes, the predicted outcome is not a certainty but only a probability; and, consequently, any therapeutic action following the assessment is based on probabilistic prognostic reasoning. We present here a comprehensive guide summarising the basic concepts of probabilistic prognostic reasoning for psychosis prediction, illustrated by practical examples from the real world scenario of highrisk services. We hope this manuscript will be of practical utility for clinicians and clinical researchers interested in the early detection of psychosis.

\section{PROBABILISTIC PROGNOSTIC REASONING}

\section{Risk or cumulative incidence of psychosis in CHR samples}

As in any preventive approach, the main aim of an indicated prevention is a reduction in the overall morbidity or cumulative incidence of the disease. ${ }^{10}$ In case of the early detection of psychosis, the main outcome is therefore defined by the transition risk to frank psychosis from a CHR state. ${ }^{11}$ Thereby, cumulative incidence is usually defined as the probability that a particular event, such as occurrence of a particular disease, has occurred within a given period. ${ }^{12}$ Synonyms of cumulative incidence include incidence proportion and risk or probability of developing psychosis. It is usually calculated by the following proportion, assuming that all patients with CHR have been followed-up:

\section{Risk of psychosis in patients with CHR $=\frac{\text { number of transitions to psychosis during a specified period }}{\text { number of patients with CHR at baseline }}$}

Such probabilities are commonly defined as the likelihood of an event with values ranging from 0 or $0 \%$ (no expected occurrence of the event in any case) to 1 or $100 \%$ (certain of the occurrence of the event in all cases). However, as the risk of psychosis onset in patients with CHR is the probability that psychosis will occur within a defined period of time and, consequently, heavily depends on the observation time, risk of psychosis in patients with CHR always has to be referred to in terms of a specified follow-up period.

Example A: No consideration of potential dropouts

One hundred patients with CHR were followed-up, first for 1 year and then for another 2, that is, altogether 3 years. At the end of 1 year, 15 patients had developed psychosis.

$\rightarrow 1$-year risk of psychosis $=15 / 100=0.15$

At the end of 3 years, 14 further patients had developed psychosis, cumulating to altogether 29 cases with a transition to psychosis within 3 years.

$\rightarrow 3$-year risk of psychosis $=(15+14) / 100=0.29$ 


\section{Person-time or incidence rate of psychosis in CHR}

Other than risk or cumulative incidence of psychosis that assumes that the outcome of all patients with CHR at the end of the follow-up period is known, the incidence rate or person-time rate is a measure that incorporates the true observation time directly into the denominator. Thus, it can account for the unfortunately likely persons who dropout during longer observation periods. As for the proportion used to compute the cumulative incidence, the numerator of the person-time rate is the number of transitions to psychosis during the follow-up time. However, the denominator is not the baseline number of patients with $\mathrm{CHR}$ but the total of the observation time (commonly given in years) of each patients with $\mathrm{CHR}$ (see example $\mathrm{B}$ below).

\section{Example B. Consideration of potential dropouts}

Again, 100 patients with CHR were followed up annually for 3 years. After 1 year, 15 had developed psychosis but 10 were lost to follow-up. After 2 years, 5 had developed psychosis and 5 were additionally lost to follow-up. After 3 years, another 9 had developed psychosis and 10 were lost to follow-up. To calculate the person-time rate of psychosis, we will assume that patients with CHR with psychosis onset and those lost to follow-up were disease-free for 6 months and thus contribute 0.5 years to the denominator.

$\rightarrow$ Person-time rate of psychosis $=(15+5+9) /(75+0.5 \times 15$

$+0.5 \times 10)+(65+0.5 \times 5+0.5 \times 5)+(55+0.5 \times 9+0.5 \times 10)$

$=29 / 222=0.13$ per persons-years of follow-up

Thus, the 3-year person time or incidence rate of psychosis with consideration of individual observation times $(=3 \times 0.13=0.39)$ is considerably higher than the 3-year cumulative incidence of Example $A(=0.29)$. This leads to the question, which measure (person-time rate vs cumulative incidence) should followed in clinical practice. Since the cumulative incidence ignores patients lost to follow-up, and conservatively assumes that they remained disease-free over the years, it might actually underestimate the true probability of psychosis transition in CHR samples. Indeed, there is evidence that about $23 \%$ of patients with CHR who disengage from CHR services (drop-outs) will later develop psychosis. ${ }^{13}$ Thus, in practice, the incidence rate relating to person-years should be preferred over the more conservative cumulative incidence or risk.

\section{Kaplan-Meier estimates of psychosis risk in patients with CHR}

An even more accurate time-to-event estimate of risk of psychosis, which also deals with incomplete observations is provided by Kaplan-Maier (survival) curves. ${ }^{14}$ A Kaplan-Maier curve is defined as the probability of surviving in a given length of time, thereby considering time in many small intervals. ${ }^{15}$ A previous meta-analysis provided a summary Kaplan-Maier estimate of psychosis risk in CHR samples (mainly by UHR criteria), and indicated that most transitions occurred within the first 2 years. ${ }^{5}$ Owing to this, it may be clinically relevant to know the exact risk of psychosis onset in patients with $\mathrm{CHR}$, given psychosis will occur during this timeframe (this will apply to most transitions although later transitions are also possible, in particular in CHR samples defined by BS criteria). ${ }^{7}$

Time to transition, given transition will occur in the first 2 years, is depicted in figure 1. Such a figure-or rather such a set of figures, considering the impact of type of risk criteria, age, referral source and possibly other patient characteristics - might be of clinical interest because they might be practically used by CHR clinicians to optimise the timing of their prognostic assessments and treatments accordingly leg, more frequent CHR reassessments in the first 2 years to not miss the chance to adapt treatment to possible symptom exacerbations ${ }^{16}$ ).

\section{Measures of prognostic accuracy in CHR samples}

Next to these risk estimates relating predominately to CHR populations, risk estimates that rather relate to CHR assessments, that is, a test's accuracy measures, are often used in diagnostic decision-making. ${ }^{17}$ These include a range of measures derived from a $2 \times 2$ contingency table, cross-classifying the binary result of a CHR assessment and the binary result of the follow-up gold standard assessment of psychosis (table 1), that will be discussed in the following.

\section{Sensitivity and specificity of CHR assessment}

In simple terms, the sensitivity of the CHR assessment is defined as the proportion of patients developing psychosis who have a positive $\mathrm{CHR}$ assessment result $(\mathrm{CHR}+)$. The specificity of a test is the proportion of patients not developing psychosis who have a negative CHR assessment result (CHR-).

\section{Example C: Sensitivity and specificity of a CHR assessment}

$$
\begin{aligned}
& \text { A total of } 150 \text { patients seeking help at a CHR service undergo } \\
& \text { CHR assessment. One hundred of them are deemed at CHR+ } \\
& \text { and of these } 29 \text { will develop psychosis at } 3 \text { years (CHR+T). } \\
& \text { Among the } 50 \text { not considered at risk, that is, CHR-, } 2 \text { will } \\
& \text { develop psychosis at } 3 \text { years (CHR-T) and } 48 \text { will not (CHR } \\
& - \text { NT). Thus, sensitivity and specificity of the CHR assessment } \\
& \text { at 3-year follow-up are: } \\
& \rightarrow \text { Sensitivity }=(\text { CHR }+\mathrm{T}) \times 100 /(\mathrm{CHR}+\mathrm{T}+\mathrm{CHR}-\mathrm{T})=29 \times 100 / \\
& (29+2)=93.5 \% \\
& \rightarrow \text { Specificity }=(\mathrm{CHR}-\mathrm{NT}) \times 100 /(\mathrm{CHR}-\mathrm{NT}+\mathrm{CHR}+\mathrm{NT}) \\
& \quad=48 \times 100 /(48+71)=40.3 \%
\end{aligned}
$$

A perfect assessment would have 100\% sensitivity and 100\% specificity, yet in the likely absence of such perfect values, no clear guidelines to weigh sensitivity against specificity exist but only rules-of-thumb. ${ }^{18}$ For example, Sackett ${ }^{19}$ suggested to use the test with the highest sensitivity for 'ruling out' and that with the highest specificity for 'ruling in' the disease, irrespective of the number of false-positive or falsenegative classifications. In contrast, balancing severity of the disorder against risk associated with treatment, McNeil et al ${ }^{20}$ suggested to use highly sensitive tests, even if of little specificity, when the disorder is

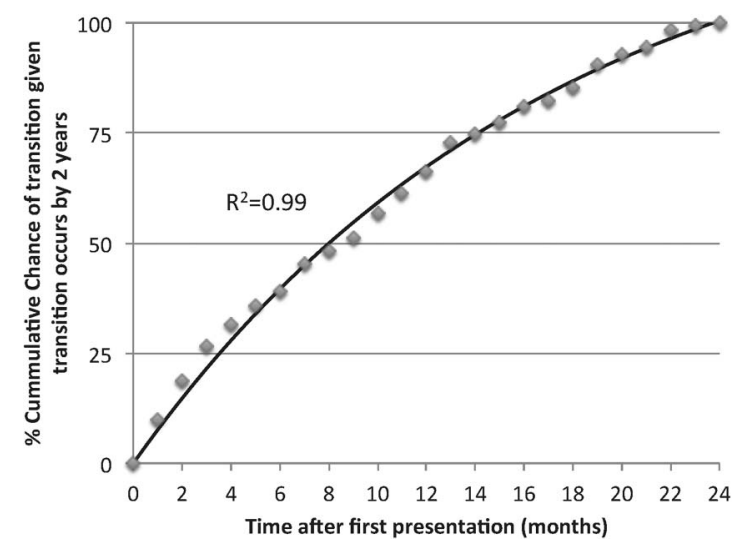

Figure 1 Cumulative chance of transition given transition occurs by 2 years. As many studies assessed transition at 2 years of follow-up, ${ }^{5}$ we plotted cumulative estimate of transition, given transition occurs by 2 years after presentation. The regression line (solid line) closely fitted the data points $(R 2=0.995 ; y=133(1-e-0.06 x))$ and indicates that, among ultra-high risk individuals progressing to psychosis in the first 2 years, $25 \%$ will develop the disorder by 106 days and $50 \%$ by 240 days. 
Table 1 Measures of prognostic accuracy that can be calculated from a $2 \times 2$ table showing the cross-classification of a CHR assessment and subsequent gold standard assessment of psychosis

\begin{tabular}{|c|c|c|c|}
\hline & \multicolumn{3}{|c|}{ Population seeking help at CHR services } \\
\hline & \multicolumn{3}{|c|}{ Psychotic disorder as defined with the gold standard ICD/DSM } \\
\hline & & Developing psychosis & Not developing psychosis \\
\hline CHR assessment outcome & $\begin{array}{l}\text { At Risk (CHR+) } \\
\text { Not At Risk (CHR-) }\end{array}$ & $\begin{array}{l}\text { CHR + T (true positive) } \\
\text { CHR- T (false negative, type II error) }\end{array}$ & $\begin{array}{l}\text { CHR + NT (false positive, type I error) } \\
\text { CHR- NT (true negative) }\end{array}$ \\
\hline Pretest probability (x-axis in figure 2 ) & \multicolumn{3}{|c|}{$\begin{array}{l}\text { The probability of developing psychosis before the CHR assessment, also called the prevalence of the disease: (true } \\
\text { positive +false negative)/(true positive }+ \text { false postive }+ \text { false negative + true negative) }\end{array}$} \\
\hline Sensitivity & \multicolumn{3}{|c|}{$\begin{array}{l}\text { Proportion of patients who will develop the psychosis who will have a positive CHR assessment result: true } \\
\text { positive/(true positive + false negative }\end{array}$} \\
\hline Specificity & \multicolumn{3}{|c|}{$\begin{array}{l}\text { The proportion of patients without the development of psychosis who will have a negative CHR assessment result: } \\
\text { true negative/(true negative }+ \text { false positive) }\end{array}$} \\
\hline Positive Predictive Value (PPV) & \multicolumn{3}{|c|}{$\begin{array}{l}\text { The proportion of patients with a positive CHR assessment result who actually have developed psychosis: true } \\
\text { positive/(true positive + false positive) }\end{array}$} \\
\hline Negative predictive value (NPV) & \multicolumn{3}{|c|}{$\begin{array}{l}\text { The proportion of patients with a negative CHR assessment result who do not have actually developed psychosis: } \\
\text { true negative/(true negative + false negative) }\end{array}$} \\
\hline Accuracy & \multicolumn{3}{|c|}{$\begin{array}{l}\text { The proportion of true results among the total number of } \mathrm{CHR} \text { patients assessed: (true positive }+ \text { true negative)/(true } \\
\text { positive }+ \text { false positive }+ \text { true negative }+ \text { false negative) }\end{array}$} \\
\hline $\begin{array}{l}\text { Positive likelihood ratio } \\
\text { LR+ (legend to figure 2) }\end{array}$ & \multicolumn{3}{|c|}{$\begin{array}{l}\text { The probability of a CHR patient who develop psychosis testing positive at the CHR assessment divided by the } \\
\text { probability of a person who does not develop the psychosis testing positive at the CHR assessment: sensitivity/ } \\
\text { (1-specificity) }\end{array}$} \\
\hline Negative likelihood ratio LR- (legend to figure 2) & \multicolumn{3}{|c|}{$\begin{array}{l}\text { The probability of a patient who develop psychosis testing negative at the CHR assessment divided by the } \\
\text { probability of a patient who does not develop psychosis testing negative at the CHR assessment: (1-sensitivity)/ } \\
\text { specificity }\end{array}$} \\
\hline Post-test positive probability (red line in figure 2) & \multicolumn{3}{|c|}{$\begin{array}{l}\text { The individual probability of developing the disease given a positive prognostic test result: Pretest } \\
\text { probability } \times \text { Sensitivity } /((\text { Pretest probability } \times \text { Sensitivity })+(1-\text { Pretest probability }) \times(1-\text { Specificity }))\end{array}$} \\
\hline Post-test negative probability (green line in figure 2) & \multicolumn{3}{|c|}{$\begin{array}{l}\text { The individual probability of developing the disease given a negative prognostic test result: (1-Pretest } \\
\text { probability }) \times \text { Specificity } /(1-\text { Pretest probability }) \times \text { Specificity })+ \text { Pretest probability } \times(1-\text { Sensitivity }))\end{array}$} \\
\hline
\end{tabular}

APS, attenuated psychosis symptoms; BIPS, brief intermittent psychotic symptoms; BLIPS, brief limited intermittent psychotic symptoms; BS, basic symptoms. Adapted from; ${ }^{8}$ CHR, clinical high risk (UHR and/or BS); DSM, Diagnostic and Statistical Manual; GRFD, genetic risk and deterioration syndrome; ICD, International Classification of Diseases; UHR, ultra high risk (including BLIPS/BIPS and/or APS and/or GRFD).

severe but its treatment benign and safe; and highly specific tests, even if of little sensitivity, when treatments carry the risk of severe side-effects and long-term consequences.

Furthermore, clinical utility of sensitivity and specificity is limited by the fact that they cannot be directly used to estimate the individual probability of developing psychosis in a patient undergoing CHR assessment. Indeed, high sensitivity and specificity do not ensure that the test will be clinically useful, nor do low sensitivity and specificity render a test useless. ${ }^{21}$ Sensitivity and specificity may also be instable and affected by factors such as sampling bias, small samples, bias in test scores or errors in selection and use of tests. ${ }^{21}$

The sensitivity and specificity of CHR assessments have recently been estimated at meta-analytical level. The prognostic sensitivity for psychosis prediction at 38 months was $96 \%$ (95\%-Cl 92\% to $98 \%$ ), while the prognostic specificity for psychosis prediction at 38 months was $47 \%(95 \%-\mathrm{Cl} 38 \%$ to $57 \%){ }^{22}$ These values indicate that CHR assessments have an outstanding ability to rule out psychosis risk and an only modest ability to rule in subsequent psychosis. ${ }^{22}$

\section{Sensitivity and specificity in ROC curves}

Sensitivity and specificity at different data points (eg, sum scores) of continuous data can be plotted in the ROC curves. ROC curves plot (1-specificity) of a test on the $x$-axis against its sensitivity on the $y$-axis. The area under this curve (AUC) represents the overall accuracy of a test, with values of 0.9-1.0 (equal to 90-100\%) considered outstanding, of 0.8-0.9 excellent and of 0.7-0.8 acceptable. ${ }^{23}$ The offdiagonal on the graph represents an AUC of 0.5 , indicating only random discrimination, that is, a test whose results are no better than tossing a coin. ROC curve for CHR assessment was reported as very good $\left(A U C=0.90 ; 95 \% \mathrm{Cl} 0.87\right.$ to 0.93). ${ }^{22}$

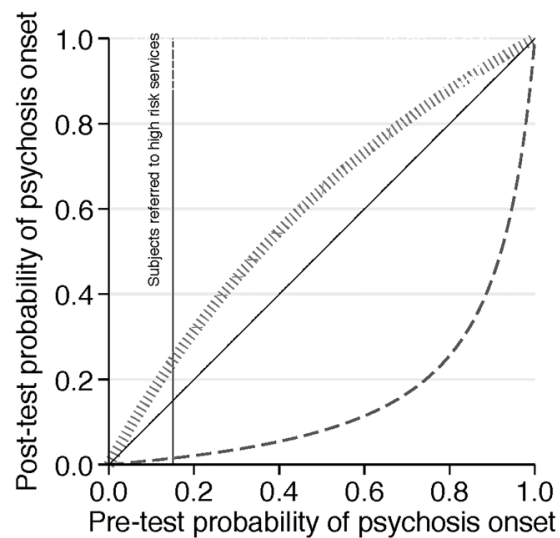

$$
\begin{aligned}
& \hline \text { IIIIII } \text { Positive CHR assessment } \\
& \text { LR+ }=1.82(95 \% \mathrm{Cl}: 1.52-2.18) \\
& \text { - }- \text { Negative CHR assessment } \\
& \text { LR- }=0.09(95 \% \mathrm{Cl}: 0.04-0.18)
\end{aligned}
$$

Figure 2 Meta-analytical probability modifying plot, illustrating the relationship between pretest probability of psychosis onset 38 months and post-test probability of psychosis risk at 38 months based on clinical high risk (CHR) psychometric assessment in patients seeking help at high-risk services, computed as the likelihood of a positive (above diagonal line in red; $L R+$ ) or negative (below diagonal line in green, LR-) CHR assessment result over the $0-1$ range of pretest probability, adapted from. ${ }^{22}$ The vertical black line indicates the average pretest probability of psychosis onset in patients referred to high-risk services $\left(15 \%\right.$ at 38 months $\left.^{8}\right)$ which yields a $26 \%$ post-test probability of psychosis onset in $\mathrm{CHR}+$ cases and a $1.56 \%$ post-test probability of psychosis onset at 38 months in CHR- cases. ${ }^{22}$ LR, likelihood ratio. 


\section{Positive and negative predictive values}

Accuracy measures that-other than sensitivity and specificity-are not considered independent of the risk of psychosis in the population to that a particular patient with CHR belongs are the positive and negative predictive values (PPV, NPV). ${ }^{24}$ The PPV is the proportion of $\mathrm{CHR}+$ patients who actually have developed psychosis $(T)$, whereas NPV is the proportion of CHR- patients who have not developed psychosis (NT). ${ }^{25}$ The PPV of CHR assessment at different time points has been reported in an earlier meta-analysis, indicating a risk of psychosis onset among $\mathrm{CHR}+$ patients between $18 \%(95 \%-\mathrm{Cl} 12 \%$ to $25 \%)$ at 6 months, and $36 \%(95 \%-\mathrm{Cl} 24 \%$ to $35 \%)$ at more than 36 months. $^{5}$ Coming back to Example C, a CHR test with $93.5 \%$ sensitivity and $40.3 \%$ specificity at 3 years, the corresponding risk of psychosis, PPV and NPV are:

- Risk of psychosis the population assessed $=(29+2) \times 100 /$ $150=20.7 \%$

- $\mathrm{PPV}=\mathrm{CHR}+\mathrm{T} \times 100 /(\mathrm{CHR}+\mathrm{T}+\mathrm{CHR}+\mathrm{NT})=29 \times 100 /(29+71)$ $=29.0 \%$

- NPV $=$ CHR $-\mathrm{NT} \times 100 /(\mathrm{CHR}-\mathrm{NT}+\mathrm{CHR}-\mathrm{T})=48 \times 100 /(48+2)$ $=96.0 \%$

Example D: Influence of the population's risk of psychosis on PPV and NPV

Let's now assume we assess CHR in a different population of 973 patients, 591 of whom will test CHR + and $382 \mathrm{CHR}-$. Among the $\mathrm{CHR}+, 29$ will develop psychosis $(\mathrm{CHR}+\mathrm{T})$ and 562 not (CHR+NT); among those CHR-, 2 would develop psychosis (CHR-T) and 380 not (CHR-NT). Sensitivity and specificity would remain the same, sensitivity $93.5 \%$ and specificity $40.3 \%$. However, the prevalence of psychosis risk in the population assessed would be lower, impacting in particular on the PPV:

$\rightarrow$ Risk of psychosis in the population $=(29+2) \times 100 / 973=3.2 \%$

$\rightarrow \mathrm{PPV}=2900 /(29+562)=4.9 \%$

$\rightarrow \mathrm{NPV}=38000 /(380+2)=99.5 \%$

Although PPV and NPV may be used to estimate the individual probabilities of developing psychosis in CHR patients, ${ }^{26}$ their clinical usefulness is restricted to populations whose prevalence of disease is similar to the one from which they were estimated. ${ }^{26}$ Recent meta-analyses indicated that the prevalence of psychosis in patients undergoing $\mathrm{CHR}$ assessment is of $15 \%$, with high heterogeneity (95\% Cl $9 \%$ to $24 \%$ ) across individual sites. ${ }^{8}$

\section{Bayes' theorem and predictive ability of CHR assessment}

Ultimately, the value of a test will depend on its ability to alter a pretest probability of a target condition into a post-test probability that will influence a clinical management decision. ${ }^{25}$ Pretest and post-test probability of psychosis in CHR patients index an individual's likelihood of the development of psychosis before and after the CHR assessment, respectively. To be clinically useful, the results of the CHR assessment should substantially change the pretest probability of the individual patient to develop the disease, that is, significantly increase or decrease his/her post-test probability. ${ }^{8}$ These concepts are linked to Bayes' theorem that describes the probability $(P)$ of an event (eg, transition to psychosis, T), based on conditions that might be related to the event (eg, being $\mathrm{CHR}+$ ). In case of early detection, Bayes' theorem can be stated mathematically as:

$$
\mathrm{P}(\mathrm{T} \mid \mathrm{CHR}+)=\mathrm{P}(\mathrm{CHR}+\mid \mathrm{T}) \times \mathrm{P}(\mathrm{T}) / \mathrm{P}(\mathrm{CHR}+)
$$

or in words: The conditional probability of development of psychosis given a positive CHR assessment, $\mathrm{P}(\mathrm{T} \mid \mathrm{CHR}+)$, is the product of the conditional probability of $\mathrm{CHR}+$ given that psychosis develops, $\mathrm{P}(\mathrm{CHR}$ + (psychosis), and the probability of psychosis, $P(T)$, divided by the probability of a positive CHR assessment, $\mathrm{P}(\mathrm{CHR}+)$.

However, $\mathrm{P}(\mathrm{CHR}+)$ and $\mathrm{P}(\mathrm{T})$ and, consequently, their related conditional probabilities are no fixed numbers but depend on sampling biases. For example, $\mathrm{P}(\mathrm{CHR}+)$ and $\mathrm{P}(\mathrm{T})$ as well as $\mathrm{P}(\mathrm{T} \mid \mathrm{CHR}+$ ) were higher in $\mathrm{CHR}$ samples recruited from mental health professionals than in those recruited from the community. ${ }^{12}$ The same was true for predominately or exclusively adult samples compared to child and adolescent samples. ${ }^{7}$ Thus, it is not only CHR criteria that determine the post-test probability of transition to psychosis but also the recruitment and selection of samples, which might create substantial enrichment in risk. ${ }^{8}$ These concepts are illustrated in the probability modifying plot (figure 2) which plots pretest probability of developing psychosis before the CHR assessment on the $x$-axis against the post-test probability of developing psychosis after CHR assessment on the y-axis. The post-test probability in turns can be positive $\mathrm{P}(\mathrm{T} \mid \mathrm{CHR}+$ ) or negative $\mathrm{P}(\mathrm{T} \mid \mathrm{CHR}-)$. The positive and negative posterior probability curves visually show that $\mathrm{CHR}$ assessment has an excellent ability to rule out psychosis, and a modest ability to rule in.

\section{Likelihood ratios}

Several authors have suggested the use of positive and negative likelihood ratios $(L R+, L R-)$ instead of sensitivity and specificity ${ }^{24}$ to better account for differences in the quality between the two and for pretest probabilities. LRs combine the estimates of sensitivity and specificity and offer the advantage of existing guidelines for their quality assessment. $^{27}$ Thereby, LR+ (=Sensitivity/(1-Specificity)) (table 1) gives the factor by that the pretest probability increases given a positive test result, while $L R-(=(1-$ Sensitivity $) /$ Specificity $)$ gives the fraction to that the post-test probability decreases given a negative test result.

As a rough guide, $\mathrm{LR}+>10$ and $\mathrm{LR}-<0.1$ generate large and often diagnostically conclusive changes from pretest to post-test probability; $L R+$ of $5-10$ and $L R-$ of $0.1-0.2$ produce moderate shifts in probability; $\mathrm{LR}+$ of $2-5$ and $\mathrm{LR}-$ of $0.2-0.5$ result in small (but sometimes important) changes in probability, while $\mathrm{LR}+<2$ and $\mathrm{LR}->0.5$ change probability to a very small (and rarely important) degree. ${ }^{24} \mathrm{~A}$ graphical presentation of LRs with the probability modifying plot (figure 2) can improve the accuracy of estimated post-test disease probability in clinical practice. $^{17}$

In a previous meta-analysis, we have shown that current CHR assessment has an excellent ability to rule out psychosis $(\mathrm{LR}-=0.09)$, at an expense of their ability to rule in psychosis $(L R+=1.82)^{22}$ and these LRs are reported in figure 2. In fact, given a pretest probability of $15 \%$ in patients referred to high-risk services, ${ }^{8}$ the post-test probability of psychosis onset at 38 months, given a CHR + assessment is of $26 \%$ $(95 \% \mathrm{Cl} 23 \% \text { to } 30 \%)^{22}$ and given a CHR- assessment is of $1.56 \%$ (95\% Cl $0.7 \%$ to $2.42 \%)^{22}$

\section{Assessing psychosis risk in clinical practice: the potentials and pitfalls of real-life application}

While LRs thereby offer a good tool for the probabilistic assessment of individual risk, they were shown to be the least well understood accuracy measures by clinicians. ${ }^{17} 28$ Yet, probabilistic reasoning makes considerable demands on the clinician even beyond understanding of LRs, for example, estimating an individual's pretest risk of psychosis onset based on available, often heterogeneous and fragmented information on population estimates, as roughly illustrated by table 2. However, probabilistic reasoning in CHR has already been very useful in advising clinicians on the use of CHR assessment. As shown in table 2, probabilistic reasoning shows that $\mathrm{CHR}$ assessment should be restricted to populations seeking help at high-risk services (case 3) only, to avoid small and negligible PPVs and NPVs (cases 1 and 2). Unfortunately, the pretest probability of psychosis onset in different subsamples (eg, migrants, 
Table 2 Description of three hypothetical cases, from real-world scenarios, illustrating the impact of different pretest probabilities of psychosis onset related to potential sampling bias on estimated post-test probabilities of psychosis onset after CHR assessment, computed using the LRs indicated in figure $2^{22}$

\begin{tabular}{llll}
\hline & Case 1 & Case 2 & Case 3 \\
\hline Population & General population & Genetic high-risk population & Mental health service users \\
Setting & School & Relative's self-support group & CHR service \\
Gender & Male & Female & Male \\
Age & 21 years & 19 years & 20 years \\
Ethnicity & Black Caribbean & White & White \\
Family history of psychosis & No & Mother diagnosed with schizophrenia & No \\
Estimated 3-year risk of & $0.27 \%$ (assuming an annual incidence of & $4 \%$ (assuming a 3-year risk of psychosis in & $15 \%$ (assuming a 3-year risk for \\
psychosis of population $\approx$ & schizophrenia in the general population of & offspring of mothers with schizophrenia as & psychosis of 15\% in help-seeking \\
3-year pretest probability & Black Caribbean migrants of 0.091\% ${ }^{30}$ ) & estimated from the Kaplan-Meier, figure 2 from ${ }^{31}$ ) & samples referred to CHR services ${ }^{8}$ ) \\
$\begin{array}{l}\text { Assessment } \\
\text { 3-year post-test probability }\end{array}$ & Interview (CHR assessment) & Interview (CHR assessment) & Interview (CHR assessment) \\
in case of positive result & $0.49 \%$ & $7.05 \%$ & $26.00 \%$ \\
(LR+ 1.82) & & & \\
3-year post-test probability & $0.02 \%$ & $0.38 \%$ & $1.56 \%$ \\
$\begin{array}{l}\text { in case of negative result } \\
\text { (LR- 0.09) }\end{array}$ & & &
\end{tabular}

CHR, clinical high risk; LR, likelihood ratio.

substance users) of patients seeking help at high-risk services is still not unknown. Thus, future research should overcome the limited pretest probability data ${ }^{29}$ by providing CHR clinicians with reliable and simple, yet not over-simplistic information on pretest probabilities in different samples seeking help at high-risk services and LRs that is presented in a well-applicable, for example, graphic form and with good guidelines for their use.

\section{CONCLUSIONS}

The current reviews illustrated the basic concepts that are necessary for probabilistic reasoning in the CHR state. Understanding basic measures used for prognostic probabilistic reasoning is a prerequisite for successfully improving the prediction of psychosis in clinical practice. ${ }^{8}$ Future refinement of these measures for CHR patients may actually influence risk management, especially as regards initiating or withholding treatment. ${ }^{17}$

\section{Competing interests None declared.}

Provenance and peer review Not commissioned; internally peer reviewed.

doi:10.1136/eb-2015-102295

Received 11 December 2015; Revised 17 December 2015;

Accepted 22 December 2015

\section{REFERENCES}

1. Kirkbride JB, Errazuriz A, Croudace TJ, et al. Incidence of schizophrenia and other psychoses in England, 1950-2009: a systematic review and meta-analyses. PLoS ONE 2012;7:e31660

2. Penttila M, Jaaskelainen $\mathrm{E}$, Hirvonen $\mathrm{N}$, et al. Duration of untreated psychosis as predictor of long-term outcome in schizophrenia: systematic review and meta-analysis. Br J Psychiatry 2014;205:88-94.

3. Valmaggia LR, Byrne M, Day F, et al. Duration of untreated psychosis and need for admission in patients who engage with mental health services in the prodromal phase. Br J Psychiatry 2015;207:130-4.

4. Fusar-Poli $\mathbf{P}$, Borgwardt $\mathrm{S}$, Bechdolf $\mathrm{A}$, et al. The psychosis high-risk state: a comprehensive state-of-the-art review. JAMA Psychiatry 2013;70:107-20.

5. Fusar-Poli P, Bonoldi I, Yung AR, et al. Predicting psychosis: meta-analysis of transition outcomes in individuals at high clinical risk. Arch Gen Psychiatry 2012:69:220-9.

6. Kempton M, Bonoldi I, Valmaggia L, et al. Speed of psychosis progression in people at ultra high clinical risk: a complementary meta-analysis. JAMA Psychiatry 2015;72:622-3.

7. Schultze-Lutter F, Michel C, Schmidt SJ, et al. EPA guidance on the early detection of clinical high risk states of psychoses. Eur Psychiatry 2015;30 405-16
8. Fusar-Poli $\mathbf{P}$, Schultze-Lutter F, Cappucciati M, et al. The dark side of the moon: meta-analytical impact of recruitment strategies on risk enrichment in the clinical high-risk state for psychosis. Schizophr Bull 2015. 20 Nov 2015. pii: sbv162. [Epub ahead of print].

9. Schimmelmann B, Michel C, Martz-Irngartinger $A$, et al. Age matters in the prevalence and clinical significance of ultra-high-risk for psychosis symptoms and criteria in the general population: findings from the BEAR and BEARS-Kid studies. World Psychiatry 2015;14:189-97.

10. WHO. Prevention of mental disorders. Effective interventions and policy options. Geneva: Department of Mental Health and Substance Abuse, 2004.

11. Fusar-Poli $\mathbf{P}$, Van Os J. Lost in transition: setting psychosis threshold in prodromal research. Acta Psychiatr Scand 2013;127:248-52.

12. Dodge $\mathbf{Y}$. The Oxford dictionary of statistical terms. 6th edn (1 Aug 2006). Oxford: OUP, 2003.

13. Green CE, McGuire PK, Ashworth M, et al. Outreach and Support in South London (OASIS). Outcomes of non-attenders to a service for people at high risk of psychosis: the case for a more assertive approach to assessment. Psychol Med 2011:41:243-50.

14. Kaplan E, Meier P. Nonparametric estimation from incomplete observations. J Amer Statist Assn 1958;53:457-81.

15. Goel MK, Khanna P, Kishore J. Understanding survival analysis: Kaplan-Meier estimate. Int J Ayurveda Res 2010:1:274-8.

16. Schmidt SJ, Schultze-Lutter F, Schimmelmann BG, et al. EPA guidance on the early intervention in clinical high risk states of psychoses. Eur Psychiatry 2015;30:388-404.

17. Whiting PF, Davenport C, Jameson C, et al. How well do health professionals interpret diagnostic information? A systematic review. BMJ Open 2015;5:e008155.

18. Boyko EJ. Ruling out or ruling in disease with the most sensitive or specific diagnostic test: short cut or wrong turn? Med Decis Making 1994;14:175-9.

19. Sackett DL. Clinical reality, binary models, babies and bath water. J Clin Epidemiol 1991:44:217-19.

20. McNeil BJ, Keller E, Adelstein SJ. Primer on certain elements of medical decision making. N Engl J Med 1975;293:211-15.

21. Meehl PE, Rosen A. Antecedent probability and the efficiency of psychometric signs, patterns, or cutting scores. Psychol Bull 1955;52:194-216.

22. Fusar-Poli $\mathbf{P}$, Cappucciati $\mathrm{M}$, Rutigliano $\mathrm{G}$, et al. At risk or not at risk? Meta-analysis of the prognostic accuracy of psychometric interviews for psychosis prediction. World Psychiatry 2015;14:322-32.

23. Hosmer W, Lemeshow S. Applied survival analysis: regression modeling of time to event data. New York, NY: Wiley \& Sons, 1999.

24. Altman DG. Some common problems in medical research. In: Altman DG, ed. Practical statistics for medical research. New York, NY: Chapman and Hall, 1991:396-438.

25. Florkowski CM. Sensitivity, specificity, receiver-operating characteristic (ROC) curves and likelihood ratios: communicating the performance of diagnostic tests. Clin Biochem Rev 2008;29(Suppl 1):S83-87.

26. Akobeng AK. Understanding diagnostic tests 1: sensitivity, specificity and predictive values. Acta Paediatr 2007;96:338-41.

27. Jaeschke R, Guyatt G, Sackett DL. Users' guides to the medical literature. III. How to use an article about a diagnostic test. A. Are the results of the study valid? Evidence-Based Medicine Working Group. JAMA 1994;271:389-91. 
28. Srinivasan $\mathbf{P}$ Westover MB, Bianchi MT. Propagation of uncertainty in Bayesian diagnostic test interpretation. South Med J 2012;105

452-9.

29. Hall S, Phang SH, Schaefer JP, et al. Estimation of post-test probabilities by residents: Bayesian reasoning versus heuristics? Adv Health Sci Educ Theory Pract 2014;19:393-402.
30. Coid JW, Kirkbride JB, Barker D, et al. Raised incidence rates of all psychoses among migrant groups: findings from the East London first episode psychosis study. Arch Gen Psychiatry 2008;65:1250-8.

31. Niemi LT, Suvisaari JM, Haukka JK, et al. Cumulative incidence of mental disorders among offspring of mothers with psychotic disorder. Results from the Helsinki High-Risk Study. Br J Psychiatry 2004;185:11-17. 